\title{
APPENDIX GRAIN SIZE ANALYSES, LEG 15
}

\author{
Gerald W. Bode, Scripps Institution of Oceanography, La Jolla, California
}

Sand-silt-clay distribution was determined using the standard sieving and pipette analysis. The sediment was dried at $105^{\circ}$ to $110^{\circ} \mathrm{C}$ and then dispersed in a Calgon solution. The sand fraction was removed by wet sieving using a $63 \mu$ sieve, the fines being processed by standard pipette analysis. A description of the theory and procedures can be found in Boyce (1972) and Bader, Gerard et al. (1970).

\section{REFERENCES}

Bader, R. G., Gerard, R. D. et al., 1970. Initial Reports of the Deep Sea Drilling Project, Volume IV. Washington (U. S. Government Printing Office), 745 p.

Boyce, R. E., 1972. Grain size analyses, Leg 9: Initial Reports of the Deep Sea Drilling Project, Volume IX. Washington (U.S. Government Printing Office), p. $779-816$.

TABLE 1

Grain Size Determinations, Leg 15

\begin{tabular}{|c|c|c|c|c|c|}
\hline $\begin{array}{l}\text { Core Section } \\
\text { Top of } \\
\text { Interval } \\
(\mathrm{cm})\end{array}$ & $\begin{array}{l}\text { Depth } \\
(\mathrm{cm})\end{array}$ & $\begin{array}{c}\text { Sand } \\
(\%)\end{array}$ & $\begin{array}{l}\text { Silt } \\
(\%)\end{array}$ & $\begin{array}{r}\text { Clay } \\
(\%)\end{array}$ & Classification \\
\hline \multicolumn{6}{|l|}{ Site 146} \\
\hline $1-3,132.0$ & 100.3 & 0.1 & 8.4 & 91.5 & Clay \\
\hline $\begin{array}{l}2-1,25.0 \\
2-2,90.0 \\
2-5,90.0 \\
2-6,109.0\end{array}$ & $\begin{array}{l}254.3 \\
256.4 \\
260.9 \\
262.6\end{array}$ & $\begin{array}{l}1.9 \\
3.5 \\
6.7 \\
6.5\end{array}$ & $\begin{array}{l}41.4 \\
39.5 \\
39.7 \\
33.1\end{array}$ & $\begin{array}{l}56.8 \\
57.0 \\
53.6 \\
60.4\end{array}$ & $\begin{array}{l}\text { Silty clay } \\
\text { Silty clay } \\
\text { Silty clay } \\
\text { Silty clay }\end{array}$ \\
\hline $6-1,102.0$ & 432.0 & 18.7 & 42.1 & 39.2 & Clayey silt \\
\hline $\begin{array}{l}8-1,34.0 \\
8-2,42.0 \\
8-2,56.0 \\
8-2,61.0\end{array}$ & $\begin{array}{l}449.3 \\
450.9 \\
451.1 \\
451.1\end{array}$ & $\begin{array}{r}0.0 \\
55.8 \\
0.0 \\
0.1\end{array}$ & $\begin{array}{l}25.7 \\
15.1 \\
12.0 \\
13.4\end{array}$ & $\begin{array}{l}74.3 \\
29.2 \\
88.0 \\
86.6\end{array}$ & $\begin{array}{l}\text { Silty clay } \\
\text { Clayey sand } \\
\text { Clay } \\
\text { Clay }\end{array}$ \\
\hline $\begin{array}{l}9-1,8.0 \\
9-2,22.0 \\
9-2,34.0\end{array}$ & $\begin{array}{l}458.1 \\
459.7 \\
459.8\end{array}$ & $\begin{array}{l}0.1 \\
0.0 \\
0.0\end{array}$ & $\begin{array}{l}12.9 \\
11.2 \\
28.8\end{array}$ & $\begin{array}{l}87.0 \\
88.7 \\
71.1\end{array}$ & $\begin{array}{l}\text { Clay } \\
\text { Clay } \\
\text { Silty clay }\end{array}$ \\
\hline $10-2,85.0$ & 469.4 & 17.5 & 32.6 & 49.9 & Silty clay \\
\hline $\begin{array}{l}11-2,27.0 \\
11-2,136.0\end{array}$ & $\begin{array}{l}477.8 \\
478.9\end{array}$ & $\begin{array}{l}0.0 \\
0.0\end{array}$ & $\begin{array}{l}16.2 \\
20.7\end{array}$ & $\begin{array}{l}83.8 \\
79.2\end{array}$ & $\begin{array}{l}\text { Clay } \\
\text { Clay }\end{array}$ \\
\hline $12-1,116.0$ & 486.2 & 0.1 & 8.4 & 91.5 & Clay \\
\hline $\begin{array}{l}13-3,55.0 \\
13-4,77.0 \\
13-5,35.0\end{array}$ & $\begin{array}{l}497.5 \\
499.3 \\
500.4\end{array}$ & $\begin{array}{l}0.0 \\
0.0 \\
0.0\end{array}$ & $\begin{array}{l}5.4 \\
7.6 \\
8.9\end{array}$ & $\begin{array}{l}94.6 \\
92.4 \\
91.1\end{array}$ & $\begin{array}{l}\text { Clay } \\
\text { Clay } \\
\text { Clay }\end{array}$ \\
\hline $\begin{array}{l}14-1,136.0 \\
14-2,67.0 \\
14-3,88.0 \\
14-4,103.0\end{array}$ & $\begin{array}{l}504.4 \\
505.2 \\
506.9 \\
508.5\end{array}$ & $\begin{array}{l}0.0 \\
0.0 \\
0.2 \\
0.0\end{array}$ & $\begin{array}{l}12.0 \\
14.7 \\
18.3 \\
18.5\end{array}$ & $\begin{array}{l}88.0 \\
85.3 \\
81.5 \\
81.5\end{array}$ & $\begin{array}{l}\text { Clay } \\
\text { Clay } \\
\text { Clay } \\
\text { Clay }\end{array}$ \\
\hline $\begin{array}{l}15-1,147.0 \\
15-2,130.0 \\
15-3,61.0\end{array}$ & $\begin{array}{l}513.5 \\
514.8 \\
515.6\end{array}$ & $\begin{array}{l}0.1 \\
0.1 \\
0.1\end{array}$ & $\begin{array}{r}21.1 \\
8.0 \\
22.6\end{array}$ & $\begin{array}{l}78.8 \\
91.9 \\
77.3\end{array}$ & $\begin{array}{l}\text { Clay } \\
\text { Clay } \\
\text { Clay }\end{array}$ \\
\hline
\end{tabular}

TABLE 1 - Continued

\begin{tabular}{|c|c|c|c|c|c|}
\hline $\begin{array}{l}\text { Core, Section, } \\
\text { Top of } \\
\text { Interval } \\
\text { (cm) }\end{array}$ & $\begin{array}{c}\text { Depth } \\
(\mathrm{cm})\end{array}$ & $\begin{array}{c}\text { Sand } \\
(\%)\end{array}$ & $\begin{array}{l}\text { Silt } \\
(\%)\end{array}$ & $\begin{array}{c}\text { Clay } \\
(\%)\end{array}$ & Classification \\
\hline $\begin{array}{l}15-4,15.0 \\
15-5,26.0 \\
15-6,10.0\end{array}$ & $\begin{array}{l}516.7 \\
518.3 \\
519.6\end{array}$ & $\begin{array}{l}0.2 \\
1.4 \\
0.8\end{array}$ & $\begin{array}{r}9.4 \\
13.5 \\
20.2\end{array}$ & $\begin{array}{l}90.4 \\
85.1 \\
79.1\end{array}$ & $\begin{array}{l}\text { Clay } \\
\text { Clay } \\
\text { Clay }\end{array}$ \\
\hline $\begin{array}{l}16-1,113.0 \\
16-2,48.0 \\
16-5,62.0 \\
16-6,55.0\end{array}$ & $\begin{array}{l}522.1 \\
523.0 \\
527.6 \\
529.0\end{array}$ & $\begin{array}{l}0.1 \\
0.1 \\
0.3 \\
0.1\end{array}$ & $\begin{array}{r}13.5 \\
11.6 \\
8.7 \\
6.7\end{array}$ & $\begin{array}{l}86.4 \\
88.3 \\
91.0 \\
93.1\end{array}$ & $\begin{array}{l}\text { Clay } \\
\text { Clay } \\
\text { Clay } \\
\text { Clay }\end{array}$ \\
\hline $\begin{array}{l}17-1,62.0 \\
17-2,24.0 \\
17-3,126.0 \\
17-4,60.0 \\
17-5,3.0 \\
17-6,13.0\end{array}$ & $\begin{array}{l}530.6 \\
531.7 \\
534.3 \\
535.1 \\
536.0 \\
537.6\end{array}$ & $\begin{array}{l}0.1 \\
0.1 \\
0.1 \\
0.0 \\
0.7 \\
0.1\end{array}$ & $\begin{array}{r}10.5 \\
14.1 \\
10.2 \\
7.1 \\
30.7 \\
14.0\end{array}$ & $\begin{array}{l}89.4 \\
85.9 \\
89.7 \\
92.9 \\
68.6 \\
85.9\end{array}$ & $\begin{array}{l}\text { Clay } \\
\text { Clay } \\
\text { Clay } \\
\text { Clay } \\
\text { Silty clay } \\
\text { Clay }\end{array}$ \\
\hline $\begin{array}{l}18-1,63.0 \\
18-3,117.0 \\
18-4,155.0 \\
18-5,75.0 \\
18-6,86.5\end{array}$ & $\begin{array}{l}539.6 \\
543.2 \\
544.9 \\
545.8 \\
547.4\end{array}$ & $\begin{array}{l}1.4 \\
9.3 \\
0.1 \\
2.8 \\
2.0\end{array}$ & $\begin{array}{l}25.0 \\
37.0 \\
16.8 \\
25.2 \\
37.6\end{array}$ & $\begin{array}{l}73.5 \\
53.7 \\
83.1 \\
72.0 \\
60.4\end{array}$ & $\begin{array}{l}\text { Silty clay } \\
\text { Silty clay } \\
\text { Clay } \\
\text { Silty clay } \\
\text { Silty clay }\end{array}$ \\
\hline $\begin{array}{l}19-3,108.0 \\
19-4,125.0\end{array}$ & $\begin{array}{l}552.1 \\
553.8\end{array}$ & $\begin{array}{l}1.8 \\
0.1\end{array}$ & $\begin{array}{l}34.5 \\
18.0\end{array}$ & $\begin{array}{l}63.7 \\
81.9\end{array}$ & $\begin{array}{l}\text { Silty clay } \\
\text { Clay }\end{array}$ \\
\hline $\begin{array}{l}20-1,114.0 \\
20-3,118.0\end{array}$ & $\begin{array}{l}558.1 \\
561.2\end{array}$ & $\begin{array}{l}2.2 \\
1.6\end{array}$ & $\begin{array}{l}27.7 \\
29.2\end{array}$ & $\begin{array}{l}70.2 \\
69.2\end{array}$ & $\begin{array}{l}\text { Silty clay } \\
\text { Silty clay }\end{array}$ \\
\hline $22-1,114.0$ & 576.1 & 1.0 & 33.9 & 65.1 & Silty clay \\
\hline $23-1,133.0$ & 585.3 & 0.2 & 22.5 & 77.3 & Clay \\
\hline $24-1,130.0$ & 594.3 & 0.4 & 20.0 & 79.6 & Clay \\
\hline $26-1,96.0$ & 612.0 & 8.2 & 34.8 & 56.9 & Silty clay \\
\hline $\begin{array}{l}30-3,74.0 \\
30-4,10.0\end{array}$ & $\begin{array}{l}650.7 \\
651.6\end{array}$ & $\begin{array}{r}12.8 \\
7.9\end{array}$ & $\begin{array}{l}26.9 \\
35.3\end{array}$ & $\begin{array}{l}60.2 \\
56.7\end{array}$ & $\begin{array}{l}\text { Silty clay } \\
\text { Silty clay }\end{array}$ \\
\hline $31-3,23.0$ & 659.2 & 5.6 & 58.7 & 35.7 & Clayey silt \\
\hline \multicolumn{6}{|l|}{ Site $146 \mathrm{~A}$} \\
\hline $1-7,120.0$ & 49.2 & 9.2 & 23.1 & 67.6 & Silty clay \\
\hline $\begin{array}{l}3-2,102.0 \\
3-3,136.0\end{array}$ & $\begin{array}{r}98.5 \\
100.4\end{array}$ & $\begin{array}{l}0.0 \\
0.0\end{array}$ & $\begin{array}{l}9.7 \\
7.2\end{array}$ & $\begin{array}{l}90.2 \\
92.7\end{array}$ & $\begin{array}{l}\text { Clay } \\
\text { Clay }\end{array}$ \\
\hline \multicolumn{6}{|l|}{ Site 147} \\
\hline $\begin{array}{l}2-1,145.0 \\
2-2,134.0 \\
2-3,80.0 \\
2-4,18.0 \\
2-4,19.0 \\
2-4,99.0\end{array}$ & $\begin{array}{l}5.5 \\
6.8 \\
7.8 \\
8.7 \\
8.7 \\
9.5\end{array}$ & $\begin{array}{l}6.4 \\
1.9 \\
1.5 \\
0.1 \\
0.5 \\
2.1\end{array}$ & $\begin{array}{l}36.8 \\
39.9 \\
41.9 \\
28.5 \\
29.9 \\
30.4\end{array}$ & $\begin{array}{l}56.8 \\
58.2 \\
56.6 \\
71.4 \\
69.5 \\
67.5\end{array}$ & $\begin{array}{l}\text { Silty clay } \\
\text { Silty clay } \\
\text { Silty clay } \\
\text { Silty clay } \\
\text { Silty clay } \\
\text { Silty clay }\end{array}$ \\
\hline $\begin{array}{l}3-1,134.0 \\
3-2,45.0 \\
3-2,109.0 \\
3-3,126.0\end{array}$ & $\begin{array}{l}15.3 \\
15.9 \\
16.6 \\
18.3\end{array}$ & $\begin{array}{l}0.7 \\
0.9 \\
0.6 \\
0.4\end{array}$ & $\begin{array}{l}33.7 \\
36.2 \\
37.0 \\
33.3\end{array}$ & $\begin{array}{l}65.6 \\
62.9 \\
62.4 \\
66.3\end{array}$ & $\begin{array}{l}\text { Silty clay } \\
\text { Silty clay } \\
\text { Silty clay } \\
\text { Silty clay }\end{array}$ \\
\hline $\begin{array}{l}4-1,78.0 \\
4-2,106.0\end{array}$ & $\begin{array}{l}23.8 \\
25.6\end{array}$ & $\begin{array}{l}0.5 \\
0.8\end{array}$ & $\begin{array}{l}38.2 \\
28.6\end{array}$ & $\begin{array}{l}61.3 \\
70.6\end{array}$ & $\begin{array}{l}\text { Silty clay } \\
\text { Silty clay }\end{array}$ \\
\hline
\end{tabular}


TABLE 1 - Continued

\begin{tabular}{|c|c|c|c|c|c|}
\hline $\begin{array}{l}\text { Core, Section, } \\
\text { Top of } \\
\text { Interval } \\
(\mathrm{cm}) \\
\end{array}$ & $\begin{array}{c}\text { Depth } \\
\text { (cm) }\end{array}$ & $\begin{array}{c}\text { Sand } \\
(\%)\end{array}$ & $\begin{array}{l}\text { Silt } \\
(\%)\end{array}$ & $\begin{array}{c}\text { Clay } \\
(\%)\end{array}$ & Classification \\
\hline $\begin{array}{l}4-3,128.0 \\
4-4,132.0 \\
4-5,146.0 \\
4-6,82.0\end{array}$ & $\begin{array}{l}27.3 \\
28.8 \\
30.5 \\
31.3\end{array}$ & $\begin{array}{l}0.6 \\
0.7 \\
1.0 \\
1.4\end{array}$ & $\begin{array}{l}36.3 \\
33.7 \\
30.9 \\
23.0\end{array}$ & $\begin{array}{l}63.1 \\
65.6 \\
68.1 \\
75.6\end{array}$ & $\begin{array}{l}\text { Silty clay } \\
\text { Silty clay } \\
\text { Silty clay } \\
\text { Clay }\end{array}$ \\
\hline $5-2,86.0$ & 34.4 & 1.5 & 24.3 & 74.2 & Silty clay \\
\hline $7-2,44.0$ & 52.9 & 2.6 & 25.5 & 71.9 & Silty clay \\
\hline $8-7,110.0$ & 70.1 & 3.3 & 23.0 & 73.7 & Silty clay \\
\hline $\begin{array}{l}9-5,62.0 \\
9-6,99.0\end{array}$ & $\begin{array}{l}75.6 \\
77.5\end{array}$ & $\begin{array}{l}2.3 \\
4.4\end{array}$ & $\begin{array}{l}30.7 \\
32.2\end{array}$ & $\begin{array}{l}67.1 \\
63.4\end{array}$ & $\begin{array}{l}\text { Silty clay } \\
\text { Silty clay }\end{array}$ \\
\hline $\begin{array}{l}10-1,93.0 \\
10-2,124.0 \\
10-4,119.0 \\
10-5,88.0\end{array}$ & $\begin{array}{l}78.9 \\
80.7 \\
83.7 \\
84.9\end{array}$ & $\begin{array}{l}1.5 \\
2.2 \\
1.2 \\
1.8\end{array}$ & $\begin{array}{l}24.2 \\
23.0 \\
34.0 \\
42.0\end{array}$ & $\begin{array}{l}74.3 \\
74.7 \\
64.8 \\
56.2\end{array}$ & $\begin{array}{l}\text { Silty clay } \\
\text { Silty clay } \\
\text { Silty clay } \\
\text { Silty clay }\end{array}$ \\
\hline $\begin{array}{l}11-3,17.0 \\
11-4,126.0 \\
11-5,104.0 \\
11-6,102.0\end{array}$ & $\begin{array}{l}91.2 \\
93.8 \\
95.0 \\
96.5\end{array}$ & $\begin{array}{l}4.0 \\
5.5 \\
6.1 \\
6.0\end{array}$ & $\begin{array}{l}23.8 \\
21.5 \\
28.4 \\
29.4\end{array}$ & $\begin{array}{l}72.3 \\
73.1 \\
65.5 \\
64.7\end{array}$ & $\begin{array}{l}\text { Silty clay } \\
\text { Silty clay } \\
\text { Silty clay } \\
\text { Silty clay }\end{array}$ \\
\hline $\begin{array}{l}12-1,88.0 \\
12-2,104.0 \\
12-3,101.0 \\
12-4,120.0\end{array}$ & $\begin{array}{c}97.9 \\
99.5 \\
101.0 \\
102.7\end{array}$ & $\begin{array}{l}3.1 \\
2.8 \\
2.9 \\
0.5\end{array}$ & $\begin{array}{l}24.3 \\
21.3 \\
25.9 \\
24.8\end{array}$ & $\begin{array}{l}72.6 \\
75.9 \\
71.2 \\
74.7\end{array}$ & $\begin{array}{l}\text { Silty clay } \\
\text { Clay } \\
\text { Silty clay } \\
\text { Silty clay }\end{array}$ \\
\hline $\begin{array}{l}13-1,78.0 \\
13-2,62.0 \\
13-3,57.0 \\
13-5,68.0\end{array}$ & $\begin{array}{l}106.8 \\
108.1 \\
109.6 \\
112.7\end{array}$ & $\begin{array}{l}1.3 \\
1.0 \\
1.5 \\
1.7\end{array}$ & $\begin{array}{l}20.0 \\
23.9 \\
34.2 \\
24.9\end{array}$ & $\begin{array}{l}78.7 \\
75.1 \\
64.3 \\
73.4\end{array}$ & $\begin{array}{l}\text { Clay } \\
\text { Clay } \\
\text { Silty clay } \\
\text { Silty clay }\end{array}$ \\
\hline $\begin{array}{l}14-3,101.0 \\
14-4,122.0 \\
14-5,58.0\end{array}$ & $\begin{array}{l}119.0 \\
120.7 \\
121.6\end{array}$ & $\begin{array}{l}4.2 \\
1.9 \\
1.5\end{array}$ & $\begin{array}{l}29.6 \\
25.0 \\
32.1\end{array}$ & $\begin{array}{l}66.2 \\
73.1 \\
66.4\end{array}$ & $\begin{array}{l}\text { Silty clay } \\
\text { Silty clay } \\
\text { Silty clay }\end{array}$ \\
\hline $\begin{array}{l}15-2,126.0 \\
15-5,126.0\end{array}$ & $\begin{array}{l}126.8 \\
131.3\end{array}$ & $\begin{array}{l}2.6 \\
0.9\end{array}$ & $\begin{array}{l}19.5 \\
27.0\end{array}$ & $\begin{array}{l}77.9 \\
72.1\end{array}$ & $\begin{array}{l}\text { Clay } \\
\text { Silty clay }\end{array}$ \\
\hline $\begin{array}{l}17-2,30.0 \\
17-3,99.0 \\
17-5,86.0 \\
17-5,170.0\end{array}$ & $\begin{array}{l}145.8 \\
148.0 \\
150.9 \\
151.7\end{array}$ & $\begin{array}{l}2.5 \\
4.2 \\
3.1 \\
2.4\end{array}$ & $\begin{array}{l}32.3 \\
28.6 \\
34.5 \\
27.8\end{array}$ & $\begin{array}{l}65.2 \\
67.2 \\
62.4 \\
69.9\end{array}$ & $\begin{array}{l}\text { Silty clay } \\
\text { Silty clay } \\
\text { Silty clay } \\
\text { Silty clay }\end{array}$ \\
\hline $\begin{array}{l}18-1,60.0 \\
18-2,82.0 \\
18-3,132.0 \\
18-4,112.0 \\
18-5,120.0 \\
18-6,128.0\end{array}$ & $\begin{array}{l}153.6 \\
155.3 \\
157.3 \\
158.6 \\
160.2 \\
161.8\end{array}$ & $\begin{array}{l}6.4 \\
3.1 \\
1.5 \\
1.6 \\
1.8 \\
1.9\end{array}$ & $\begin{array}{l}31.8 \\
32.1 \\
21.4 \\
21.1 \\
36.2 \\
30.1\end{array}$ & $\begin{array}{l}61.8 \\
64.8 \\
77.1 \\
77.3 \\
61.9 \\
67.9\end{array}$ & $\begin{array}{l}\text { Silty clay } \\
\text { Silty clay } \\
\text { Clay } \\
\text { Clay } \\
\text { Silty clay } \\
\text { Silty clay }\end{array}$ \\
\hline \multicolumn{6}{|l|}{ Site $147 \mathrm{C}$} \\
\hline $\begin{array}{l}7-3,29.0 \\
7-5,37.0 \\
7-6,79.0\end{array}$ & $\begin{array}{l}173.3 \\
176.4 \\
178.3\end{array}$ & $\begin{array}{l}2.8 \\
1.5 \\
0.8\end{array}$ & $\begin{array}{l}36.3 \\
27.4 \\
39.7\end{array}$ & $\begin{array}{l}60.9 \\
71.1 \\
59.5\end{array}$ & $\begin{array}{l}\text { Silty clay } \\
\text { Silty clay } \\
\text { Silty clay }\end{array}$ \\
\hline \multicolumn{6}{|l|}{ Site 148} \\
\hline $\begin{array}{l}1-2,60.0 \\
1-3,123.0\end{array}$ & $\begin{array}{l}2.1 \\
4.2\end{array}$ & $\begin{array}{l}10.9 \\
15.2\end{array}$ & $\begin{array}{l}20.2 \\
21.2\end{array}$ & $\begin{array}{l}68.9 \\
63.6\end{array}$ & $\begin{array}{l}\text { Silty clay } \\
\text { Silty clay }\end{array}$ \\
\hline $\begin{array}{l}2-3,19.0 \\
2-4,31.0\end{array}$ & $\begin{array}{l}12.2 \\
13.8\end{array}$ & $\begin{array}{l}5.5 \\
3.4\end{array}$ & $\begin{array}{l}26.6 \\
17.5\end{array}$ & $\begin{array}{l}67.9 \\
79.0\end{array}$ & $\begin{array}{l}\text { Silty clay } \\
\text { Clay }\end{array}$ \\
\hline $\begin{array}{l}3-2,43.0 \\
3-4,55.0\end{array}$ & $\begin{array}{l}19.9 \\
23.0\end{array}$ & $\begin{array}{l}2.6 \\
1.5\end{array}$ & $\begin{array}{l}15.7 \\
11.1\end{array}$ & $\begin{array}{l}81.7 \\
87.4\end{array}$ & $\begin{array}{l}\text { Clay } \\
\text { Clay }\end{array}$ \\
\hline $4-4,112.0$ & 32.6 & 2.0 & 13.4 & 84.6 & Clay \\
\hline $6-5,155.0$ & 52.5 & 7.0 & 17.3 & 75.7 & Clay \\
\hline $\begin{array}{l}7-1,119.0 \\
7-2,119.0\end{array}$ & $\begin{array}{l}56.2 \\
57.7\end{array}$ & $\begin{array}{l}6.2 \\
2.8\end{array}$ & $\begin{array}{l}20.3 \\
26.1\end{array}$ & $\begin{array}{l}73.5 \\
71.1\end{array}$ & $\begin{array}{l}\text { Silty clay } \\
\text { Silty clay }\end{array}$ \\
\hline
\end{tabular}

TABLE 1 - Continued

\begin{tabular}{lrrrll}
\hline $\begin{array}{c}\text { Core, Section, } \\
\text { Top of } \\
\text { Interval } \\
(\mathrm{cm})\end{array}$ & $\begin{array}{r}\text { Depth } \\
(\mathrm{cm})\end{array}$ & $\begin{array}{r}\text { Sand } \\
(\%)\end{array}$ & $\begin{array}{r}\text { Silt } \\
(\%)\end{array}$ & $\begin{array}{c}\text { Clay } \\
(\%)\end{array}$ & Classification \\
\hline $9-2,74.0$ & 75.2 & 0.6 & 18.5 & 80.9 & Clay \\
$9-5,82.0$ & 79.8 & 1.4 & 19.1 & 79.5 & Clay \\
$12-3,42.0$ & 103.4 & 6.8 & 22.6 & 70.7 & Silty clay \\
$13-4,46.0$ & 114.0 & 1.2 & 24.7 & 74.2 & Silty clay \\
$15-2,22.0$ & 128.7 & 11.9 & 25.4 & 62.7 & Silty clay \\
$15-6,16.0$ & 134.7 & 1.7 & 13.4 & 84.9 & Clay \\
$16-2,20.0$ & 138.7 & 2.2 & 15.8 & 82.0 & Clay \\
$16-3,76.0$ & 140.8 & 1.9 & 17.3 & 80.7 & Clay \\
$16-4,123.0$ & 142.7 & 4.1 & 22.9 & 73.0 & Silty clay \\
$17-4,31.0$ & 150.8 & 1.3 & 18.0 & 80.7 & Clay \\
$17-5,70.0$ & 152.7 & 1.8 & 14.8 & 83.5 & Clay \\
$19-3,14.0$ & 168.1 & 0.8 & 11.3 & 87.9 & Clay \\
$20-2,14.0$ & 176.6 & 1.4 & 16.2 & 82.5 & Clay \\
$20-5,74.0$ & 181.7 & 1.9 & 15.7 & 82.3 & Clay \\
$21-3,14.0$ & 187.1 & 1.6 & 13.8 & 84.6 & Clay \\
$24-3,136.0$ & 216.4 & 0.6 & 15.6 & 83.8 & Clay \\
$27-1,135.0$ & 241.4 & 5.6 & 14.1 & 80.3 & Clay \\
$27-3,126.0$ & 244.3 & 4.6 & 19.2 & 76.2 & Clay \\
$28-1,86.0$ & 249.9 & 62.0 & 20.9 & 17.1 & Silty sand \\
$31-2,32.0$ & 268.8 & 3.8 & 32.0 & 64.2 & Silty clay \\
$31-2,57.0$ & 269.1 & 42.3 & 35.0 & 22.8 & Sand-silt-clay \\
$31-3,98.0$ & 271.0 & 25.4 & 32.7 & 41.9 & Sand-silt-clay
\end{tabular}

Site 149

\begin{tabular}{lrrrrl}
$2-1,132.0$ & 2.3 & 25.5 & 19.1 & 55.3 & Sandy clay \\
$2-2,74.0$ & 3.2 & 31.1 & 16.8 & 52.1 & Sandy clay \\
$2-3,121.0$ & 5.2 & 15.7 & 15.8 & 68.5 & Silty clay \\
$2-4,93.0$ & 6.4 & 18.7 & 18.2 & 63.1 & Sandy clay \\
$2-5,87.0$ & 7.9 & 22.2 & 18.5 & 59.3 & Sandy clay \\
$2-6,86.0$ & 9.4 & 10.2 & 21.4 & 68.4 & Silty clay \\
$3-2,92.0$ & 12.4 & 24.2 & 18.1 & 57.7 & Sandy clay \\
$3-3,124.0$ & 14.2 & 22.0 & 18.4 & 59.6 & Sandy clay \\
$3-4,122.0$ & 15.7 & 20.3 & 16.0 & 63.7 & Sandy clay \\
$3-5,74.0$ & 16.7 & 9.3 & 21.0 & 69.6 & Silty clay \\
$3-6,10.0$ & 17.6 & 8.6 & 19.9 & 71.5 & Silty clay \\
$3-6,122.0$ & 18.7 & 4.2 & 20.8 & 75.1 & Clay \\
$4-1,92.0$ & 19.9 & 20.1 & 17.1 & 62.8 & Sandy clay \\
$4-2,146.0$ & 22.0 & 11.5 & 16.0 & 72.5 & Silty clay \\
$4-4,69.0$ & 24.2 & 21.3 & 15.7 & 63.0 & Sandy clay \\
$4-4,123.0$ & 24.7 & 14.6 & 14.3 & 71.1 & Sandy clay \\
$4-5,104.0$ & 26.0 & 15.9 & 17.7 & 66.4 & Silty clay \\
$5-2,144.0$ & 30.9 & 14.2 & 18.7 & 67.1 & Silty clay \\
$5-3,46.0$ & 31.5 & 1.6 & 16.9 & 81.5 & Clay \\
$5-3,94.5$ & 31.9 & 8.6 & 15.1 & 76.3 & Clay \\
$6-2,138.0$ & 39.9 & 10.6 & 12.4 & 77.0 & Clay \\
$6-3,74.0$ & 40.7 & 15.1 & 19.3 & 65.6 & Silty clay \\
$6-5,49.0$ & 43.5 & 10.3 & 23.0 & 66.8 & Silty clay \\
$6-6,14.0$ & 44.6 & 8.7 & 16.5 & 74.8 & Silty clay \\
$7-3,148.0$ & 50.5 & 5.9 & 15.5 & 78.5 & Clay \\
$7-6,69.0$ & 54.2 & 10.7 & 18.1 & 71.1 & Silty clay \\
$8-1,27.0$ & 56.3 & 13.8 & 17.4 & 68.8 & Silty clay \\
$8-1,123.0$ & 57.2 & 1.0 & 15.0 & 84.0 & Clay \\
$8-2,134.0$ & 58.8 & 1.7 & 16.1 & 82.2 & Clay \\
$8-3,45.0$ & 59.5 & 4.3 & 18.1 & 77.6 & Clay \\
$8-4,24.0$ & 60.7 & 0.9 & 16.6 & 82.5 & Clay \\
$8-5,54.0$ & 62.5 & 13.4 & 21.8 & 64.8 & Silty clay \\
$8-6,62.0$ & 64.1 & 1.2 & 14.5 & 84.3 & Clay \\
& & & & & \\
\hline & & & & & \\
& & & & &
\end{tabular}


TABLE 1 - Continued

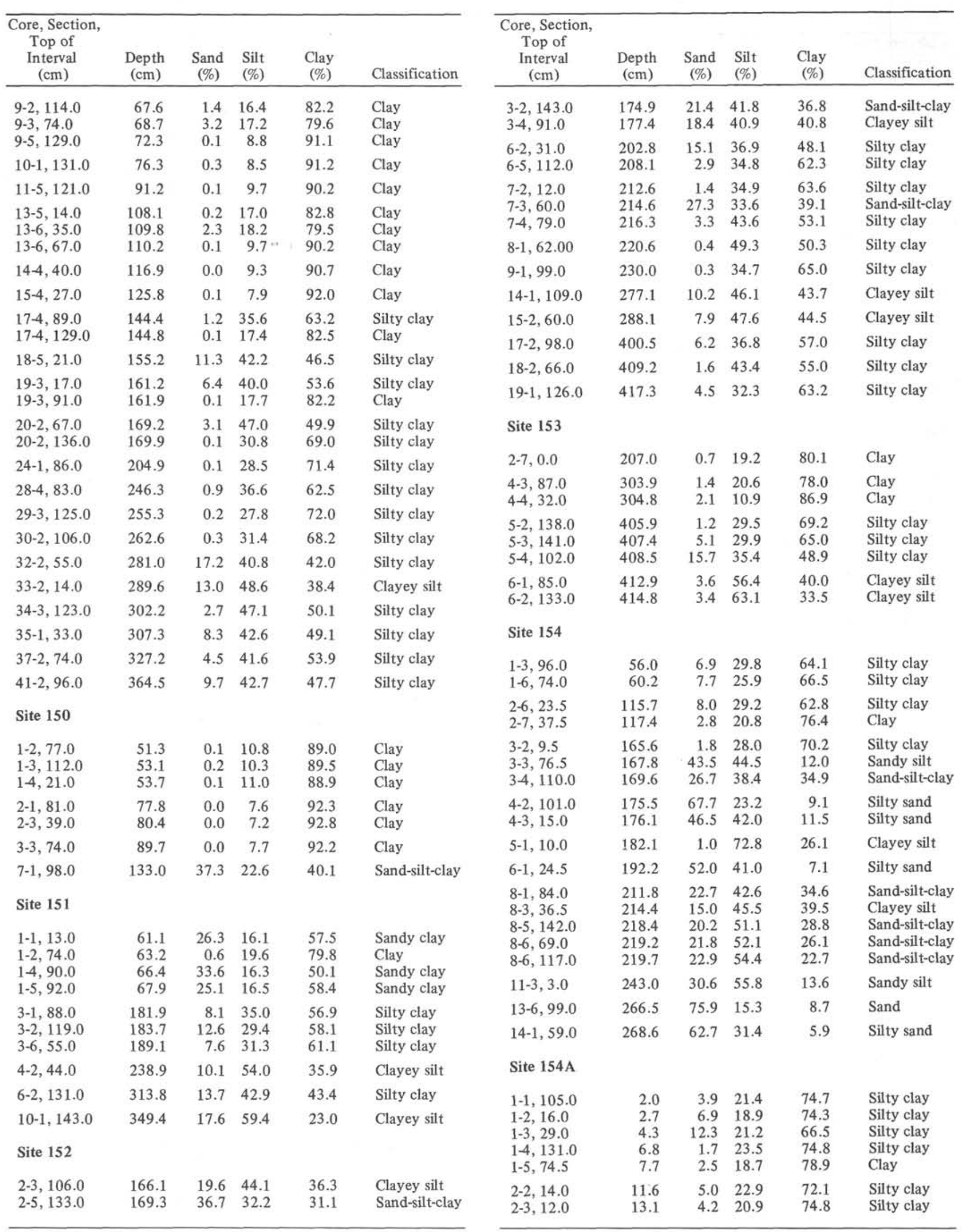

TABLE 1 - Continued

Site 154 
G. W. BODE

TABLE 1 - Continued

\begin{tabular}{lrrrrl}
\hline $\begin{array}{c}\text { Core, Section, } \\
\text { Top of } \\
\text { Interval } \\
\text { (cm) }\end{array}$ & $\begin{array}{c}\text { Depth } \\
(\mathrm{cm})\end{array}$ & $\begin{array}{c}\text { Sand } \\
(\%)\end{array}$ & $\begin{array}{c}\text { Silt } \\
(\%)\end{array}$ & $\begin{array}{c}\text { Clay } \\
(\%)\end{array}$ & Classification \\
\hline $3-1,107.0$ & 21.1 & 7.4 & 24.1 & 68.5 & Silty clay \\
$3-2,14.5$ & 21.6 & 6.8 & 26.5 & 66.7 & Silty clay \\
$3-3,18.0$ & 23.2 & 3.9 & 20.5 & 75.7 & Clay \\
$3-4,14.5$ & 24.6 & 1.5 & 20.9 & 77.6 & Clay \\
$3-5,8.0$ & 26.1 & 8.4 & 33.3 & 58.3 & Silty clay \\
$4-1,8.0$ & 29.1 & 4.3 & 20.9 & 74.7 & Silty clay \\
$4-2,90.0$ & 31.4 & 4.8 & 22.0 & 73.3 & Silty clay \\
$4-3,18.0$ & 32.2 & 4.4 & 33.3 & 62.3 & Silty clay \\
$4-4,7.5$ & 33.6 & 4.1 & 21.1 & 74.8 & Silty clay \\
$4-5,91.0$ & 35.9 & 2.1 & 21.7 & 76.3 & Clay \\
$4-6,21.0$ & 36.7 & 3.0 & 24.1 & 72.9 & Silty clay \\
$5-2,104.0$ & 41.5 & 5.5 & 20.1 & 74.3 & Silty clay \\
$7-2,98.0$ & 61.5 & 7.9 & 29.6 & 62.5 & Silty clay \\
$8-2,38.0$ & 69.9 & 5.9 & 21.7 & 72.4 & Silty clay \\
$8-5,144.0$ & 75.4 & 6.2 & 24.1 & 69.6 & Silty clay \\
$9-3,129.0$ & 82.3 & 9.0 & 26.2 & 64.8 & Silty clay \\
$9-6,94.0$ & 86.4 & 2.9 & 20.5 & 76.6 & Clay \\
$10-2,100.0$ & 89.5 & 9.5 & 28.8 & 61.8 & Silty clay \\
$10-5,117.0$ & 94.2 & 5.0 & 21.1 & 73.9 & Silty clay \\
$11-3,134.0$ & 101.3 & 3.0 & 21.3 & 75.7 & Clay \\
$11-4,130.0$ & 102.8 & 5.4 & 24.6 & 70.0 & Silty clay \\
$11-6,16.0$ & 104.7 & 3.5 & 33.1 & 63.4 & Silty clay \\
\hline & & & & &
\end{tabular}

TABLE 1 - Continued

\begin{tabular}{|c|c|c|c|c|c|}
\hline $\begin{array}{l}\text { Core, Section, } \\
\text { Top of } \\
\text { Interval } \\
\text { (cm) }\end{array}$ & $\begin{array}{l}\text { Depth } \\
\text { (cm) }\end{array}$ & $\begin{array}{c}\text { Sand } \\
(\%)\end{array}$ & $\begin{array}{l}\text { Silt } \\
(\%)\end{array}$ & $\begin{array}{r}\text { Clay } \\
(\%)\end{array}$ & Classification \\
\hline $\begin{array}{l}12-2,123.0 \\
12-5,130.0\end{array}$ & $\begin{array}{l}108.7 \\
113.3\end{array}$ & $\begin{array}{l}3.9 \\
6.2\end{array}$ & $\begin{array}{l}24.6 \\
26.4\end{array}$ & $\begin{array}{l}71.4 \\
67.4\end{array}$ & $\begin{array}{l}\text { Silty clay } \\
\text { Silty clay }\end{array}$ \\
\hline $\begin{array}{l}13-3,79.0 \\
13-4,74.0 \\
13-6,43.0\end{array}$ & $\begin{array}{l}119.8 \\
121.2 \\
123.9\end{array}$ & $\begin{array}{l}3.8 \\
4.9 \\
2.6\end{array}$ & $\begin{array}{l}25.4 \\
25.9 \\
15.7\end{array}$ & $\begin{array}{l}70.8 \\
69.2 \\
81.7\end{array}$ & $\begin{array}{l}\text { Silty clay } \\
\text { Silty clay } \\
\text { Clay }\end{array}$ \\
\hline $\begin{array}{l}14-2,62.0 \\
14-3,92.0 \\
14-4,17.0 \\
14-5,19.0 \\
14-6,34.0\end{array}$ & $\begin{array}{l}127.1 \\
128.9 \\
129.7 \\
131.2 \\
132.8\end{array}$ & $\begin{array}{l}3.5 \\
4.0 \\
6.4 \\
2.7 \\
2.9\end{array}$ & $\begin{array}{l}19.4 \\
21.3 \\
35.6 \\
18.5 \\
17.9\end{array}$ & $\begin{array}{l}77.1 \\
74.7 \\
58.0 \\
78.8 \\
79.2\end{array}$ & $\begin{array}{l}\text { Clay } \\
\text { Silty clay } \\
\text { Silty clay } \\
\text { Clay } \\
\text { Clay }\end{array}$ \\
\hline $\begin{array}{l}15-3,17.0 \\
15-5,64.0\end{array}$ & $\begin{array}{l}137.2 \\
140.6\end{array}$ & $\begin{array}{l}2.6 \\
1.5\end{array}$ & $\begin{array}{l}23.7 \\
22.0\end{array}$ & $\begin{array}{l}73.6 \\
76.5\end{array}$ & $\begin{array}{l}\text { Silty clay } \\
\text { Clay }\end{array}$ \\
\hline $\begin{array}{l}16-4,14.0 \\
16-6,14.0\end{array}$ & $\begin{array}{l}148.6 \\
151.6\end{array}$ & $\begin{array}{l}0.3 \\
0.8\end{array}$ & $\begin{array}{l}23.9 \\
26.7\end{array}$ & $\begin{array}{l}75.8 \\
72.5\end{array}$ & $\begin{array}{l}\text { Clay } \\
\text { Silty clay }\end{array}$ \\
\hline $\begin{array}{l}17-1,53.0 \\
17-3,17.0 \\
17-4,107.0 \\
17-5,49.0 \\
17-6,100.0\end{array}$ & $\begin{array}{l}153.5 \\
156.2 \\
158.6 \\
159.5 \\
161.5\end{array}$ & $\begin{array}{l}23.6 \\
43.1 \\
68.2 \\
61.9 \\
51.6\end{array}$ & $\begin{array}{l}34.3 \\
36.8 \\
24.2 \\
28.0 \\
23.7\end{array}$ & $\begin{array}{r}42.0 \\
20.1 \\
7.6 \\
10.1 \\
24.7\end{array}$ & $\begin{array}{l}\text { Sand-silt-clay } \\
\text { Sand-silt-clay } \\
\text { Silty sand } \\
\text { Silty sand } \\
\text { Sand-silt-clay }\end{array}$ \\
\hline $\begin{array}{l}18-2,26.0 \\
18-3,41.0 \\
18-4,37.0\end{array}$ & $\begin{array}{l}164.8 \\
166.4 \\
167.9\end{array}$ & $\begin{array}{l}13.6 \\
36.7 \\
40.5\end{array}$ & $\begin{array}{l}77.9 \\
37.9 \\
32.5\end{array}$ & $\begin{array}{r}8.5 \\
25.3 \\
27.0\end{array}$ & $\begin{array}{l}\text { Silt } \\
\text { Sand-silt-clay } \\
\text { Sand-silt-clay }\end{array}$ \\
\hline
\end{tabular}

